\title{
KCNJ6 is Associated with Adult Alcohol Dependence and Involved in Gene $\times$ Early Life Stress Interactions in Adolescent Alcohol Drinking
}

\author{
Toni-Kim Clarke*,', Manfred Laucht ${ }^{2,3}$, Monika Ridinger ${ }^{4}$, Norbert Wodarz $^{4}$, Marcella Rietschel ${ }^{2}$, \\ Wolfgang Maier ${ }^{5}$, Mark Lathrop ${ }^{6}$, Anbarasu Lourdusamy', Ulrich S Zimmermann ${ }^{7}$, Sylvane Desrivieres' \\ and Gunter Schumann ${ }^{1,5}$
}

'Section of Addiction Biology, MRC-SGDP Centre, Institute of Psychiatry, King's College London, London, UK; '²Department of Child and Adolescent Psychiatry and Psychotherapy, Central Institute of Mental Health, Mannheim, Germany; ${ }^{3}$ Department of Psychology, Division of Clinical Psychology and Psychotherapy, University of Potsdam, Potsdam, Germany; ${ }^{4}$ Department of Psychiatry and Psychotherapy, University of Regensburg, Regensburg, Germany; ${ }^{5}$ Department of Psychiatry and Psychotherapy, University of Bonn, Bonn, Germany; ${ }^{6}$ Centre National de Genotypage, Evry, France; ${ }^{7}$ Department of Psychiatry and Psychotherapy, University Hospital Carl Gustav Carus, Technische Universität, Dresden, Germany

\begin{abstract}
Alcohol abuse and dependence have proven to be complex genetic traits that are influenced by environmental factors. Primate and human studies have shown that early life stress increases the propensity for alcohol abuse in later life. The reinforcing properties of alcohol are mediated by dopaminergic signaling; however, there is little evidence to indicate how stress alters alcohol reinforcement. KCNJ6 (the gene encoding G-protein-coupled inwardly rectifying potassium channel 2 (GIRK2)) is a brain expressed potassium channel with inhibitory effects on dopaminergic tone. The properties of GIRK2 have been shown to be enhanced by the stress peptide corticotrophin-releasing hormone. Therefore, we sought to examine the role of KCNJ6 polymorphisms in adult alcohol dependence and stress-related alcohol abuse in adolescents. We selected I I SNPs in the promoter region of KCNJ6, which were genotyped in I I 52 adult alcohol dependents and 1203 controls. One SNP, rs28360I6, was found to be associated with alcohol dependence ( $p=0.01$, false discovery rate). We then assessed rs28360 I 6 in an adolescent sample of 261 subjects, which were characterized for early life stress and adolescent hazardous drinking, defined using the Alcohol Use Disorders Identification Test (AUDIT), to examine gene-environment interactions. In the adolescent sample, the risk genotype of rs28360I 6 was significantly associated with increased AUDIT scores, but only in those individuals exposed to high levels of psychosocial stress in early life $(p=0.01)$. Our findings show that KCNJ6 is associated with alcohol dependence and may moderate the effect of early psychosocial stress on risky alcohol drinking in adolescents. We have identified a candidate gene for future studies investigating a possible functional link between the response to stress and alcohol reinforcement. Neuropsychopharmacology (20I I) 36, II42-I |48; doi: I0.1038/npp.20 I0.247; published online 9 February 20I I
\end{abstract}

Keywords: alcoholism; genetics; GIRK2; stress; gene $\times$ environment; KCNJ6

\section{INTRODUCTION}

In 2002, the WHO's World Health Report concluded that alcohol was the fifth leading cause of death and disability worldwide, accounting for $9.2 \%$ of disability-adjusted life years in the developed world (Grimm, 2008). Alcoholism is estimated to be between 50 and $60 \%$ heritable (Dick and Bierut, 2006), which suggests that both genetic and

*Correspondence: Dr T-K Clarke, Translational Research Laboratory, Department of Psychiatry, School of Medicine, University of Pennsylvania, Room 2109, 125 South 31st St Phila, Philadelphia, PA 19104, USA, Tel: + I 215746 3665, E-mail: ctoni@mail.med.upenn.edu Received I 4 September 2010 ; revised 16 November 20 I0; accepted 8 December 2010 environmental factors are almost equally contributing to produce a complex phenotype. The dopaminergic mesolimbic system is one of the main neuronal networks responsible for inducing the reinforcing effects of drugs of abuse (Robbins and Everitt, 1999, Tupala and Tiihonen, 2004). Ion channels are known to regulate dopaminergic neuronal signalling. One such family of ion channels includes the G-protein-coupled inwardly rectifying potassium channels (GIRKs). GIRKs induce the postsynaptic inhibitory tone in response to the activation of the D2, D3 dopamine receptors, although their effects are not limited to dopaminergic neurons (Kuzhikandathil et al, 1998). As the GIRK channel is opened, potassium ions permeate the cell, hyperpolarization ensues (Nicoll, 1988), and the neuronal firing rate is reduced (Werner et al, 1996). 
While dopaminergic signalling reinforces alcohol consumption, exposure to early life stress is known to promote vulnerability to alcohol abuse in later life. This concept has been extensively studied in Rhesus macaques, where it has been shown that stress during early life increases alcohol consumption in adults (Fahlke et al, 2000). An enhanced sensitivity to stress in later life is also seen in these animals, who display increased salivary cortisol in response to social isolation. Similar findings are observed in human beings with the number of adverse childhood experiences being correlated with an increased propensity for adolescent alcohol abuse (Dube et al, 2006). Despite a well-established link between early life stress and alcohol abuse in later life, there is currently no known mechanism showing how such effects are mediated. Interestingly, the efficiency of the GIRK channels has been shown to be increased by stress peptides such as corticotrophin-releasing hormone (CRH) (Beckstead et al, 2009). Inhibitory postsynaptic currents mediated by GIRK2 channels in D2-dopaminergic neurons of the mouse brain were found to be enhanced by $\mathrm{CRH}$, and this was dependent on the CRH-R1 receptor. Furthermore, protein levels of GIRKs (GIRK1 and GIRK2) have been shown to be regulated by corticosterones in the brain of rats (Muma and Beck, 1999), providing evidence that GIRK2 interacts with neuronal stress systems. GIRK2 channels therefore may establish a link between brain stress systems, dopaminergic signalling, and thus reinforcement-related behavior.

Animal studies have analyzed the effects of ethanol on GIRK2-null mice. They found that they could not establish a place preference or conditioned taste aversion for $2-3 \mathrm{~g} / \mathrm{kg}$ of ethanol in mice lacking GIRK2 channels (Hill et al, 2003), concluding that GIRK2 may be mediating the reinforcing and/or aversive motivational aspects of ethanol action. In human beings, only one study has examined GIRK2 and addictive phenotypes. A large-scale candidate gene study analyzed SNPs in 1050 nicotine-dependent individuals and 879 controls (Saccone et al, 2007). An SNP in the promoter of KCNJ6, the gene encoding GIRK2, provided one of the top signals for nicotine dependence in this study, suggesting that GIRK2 may be important in human nicotine addiction. Our approach was to explore the significance of genetic variations in the promoter of KCNJ6 in alcohol dependence in a large sample of 1152 alcoholics and 1203 controls. In a second step, we analyzed any significant GIRK2 polymorphisms from the adult sample in a separate adolescent sample drawn from an epidemiological birth cohort of children at risk for psychopathology in later life. The adolescent sample had been characterized for both hazardous alcohol drinking at age 19 years and early psychosocial adversity to examine the role of GIRK2 in mediating gene $\times$ early life stress interactions and alcohol abuse.

\section{MATERIALS AND METHODS}

\section{Subjects}

Adult patient and control samples. In all, 1152 patients were recruited from the Department of Psychiatry at Regensburg University. All patients were admitted consecutively for in-patient treatment and met the criteria of alcohol dependence according to DSM-IV. Patients were also assessed for nicotine dependence using diagnostic criteria according to the DSM-IV and ICD-10. All participants were of German descent. Parents of the participants were living in Bavaria and participants themselves were born and raised in this area. Written informed consent was obtained for all participants before the investigation. Diagnosis was assessed after alcohol withdrawal by the Composite International Diagnostic Interview (Wittchen et al, 1996) performed by trained staff who rated participants independently. Patients with a lifetime history of schizophrenia or an addiction to drugs other than tobacco or alcohol were excluded from the study.

In total, 1203 individuals were from the region of Bonn, Germany, and were recruited from 2001 to 2003 within the German National Research Project to serve as controls for genetic studies in several neuropsychiatric phenotypes. Population-based recruitment was performed in collaboration with the local consensus bureau. Participants were screened for neurological and psychiatric disorders with self-report questionnaires adapted from the German version of the inventory to diagnose depression (Kuhner, 1997); smoking and drinking were diagnosed with the Fagerström Tolerance Questionnaire (Fagerstrom, 1978) and the Alcohol Use Disorders Identification Test (AUDIT) (Bohn et al, 1995). More than $96 \%$ of the participants were of German or Western European origin as ascertained by place of birth of their grandparents. For all patients and controls, written informed consent was obtained before study participation.

Adolescent at-risk sample. Participants were members of the Mannheim Study of Children at Risk, an ongoing epidemiological cohort study examining the outcome of early risk factors from infancy into adulthood (Laucht et al, 2000). The initial sample comprised 384 children born between 1986 and 1988, of predominantly (>99.0\%) European descent. Infants were recruited from two obstetric and six children's hospitals of the Rhine-Neckar Region of Germany and were included consecutively into the sample according to a two-factorial design intended to enrich and control the status of the sample with regard to obstetric and psychosocial risks (Laucht et al, 1997). Only firstborn children with singleton births and German-speaking parents were enrolled in the study. Furthermore, children with severe physical handicaps, obvious genetic defects, or metabolic diseases were excluded. Assessments were conducted at regular intervals throughout development, most recently at age 19 years. The current investigation included 261 adolescents (119 men, 142 women) participating in the 19 -year assessment for whom genetic data on rs2836016 were available. Of the initial sample, $18(4.7 \%)$ were excluded because of severe handicaps (IQ or MQ $<70$ or neurological disorder), 87 (22.7\%) had incomplete data or were dropouts, and $18(4.7 \%)$ refused to participate in blood sampling. The study was approved by the ethics committee of the University of Heidelberg and written informed consent was obtained from all participants.

\section{Assessment}

Adolescents completed the AUDIT, a screening instrument for the detection of hazardous alcohol use, developed by the 
WHO (Bohn et al, 1995). This assessment was carried out in the adolescent sample only. The AUDIT comprises 10 items, referring to the last 12 months, by which patterns of alcohol consumption, alcohol dependence, and adverse consequences of heavy drinking are assessed. The AUDIT has shown reasonable reliability and validity in a German sample (Dybek et al, 2006). Early psychosocial adversity according to an 'enriched' family adversity index, as proposed by Rutter and Quinton (1977), was derived from a standardized parent interview conducted at the 3-month assessment. The index assesses the presence of 11 adverse family factors, covering characteristics of the parents (eg, low education), the partnership (eg, discord), and the family environment (eg, overcrowding) during a period of 1 year before birth. (Definitions of the family adversity index are shown in Supplementary Table S1.)

The current stressful life events were assessed using a modified and shortened version of the Munich Events List (Wittchen et al, 1989). The 53-item questionnaire asked about occurrence and threat of severe life events and chronic difficulties in the 4 years before the 19-year assessment. The items addressed all areas of adolescents' lives from school, and job to partner, family, parents, living conditions, legal troubles, and health problems. Several indices can be derived from the MEL, such as total index, weighted total, and chronic life events. For the current analysis, a total life event score was computed, which counted the number of life events throughout the past 4 years (range $0-28$ ). Several studies have confirmed the psychometric characteristics of the MEL (Wittchen et al, 1989).

\section{SNP Genotyping and Statistical Analysis}

SNP genotyping was performed at the Centre National de Genotypage, Paris, France and by Prevention Genetics, Marshfield, WI, USA. In our adult sample, the allelic association of SNPs with alcoholism was determined using the $\chi^{2}$ test and $p$-values were corrected for multiple comparisons using the false discovery rate (FDR) procedure (Benjamini et al, 2001) in the software package PLINK v.1.04 (Purcell et al, 2007b). Association analysis was carried out on the nicotine-dependent adult alcoholics compared with non-nicotine-dependent alcoholics using the $\chi^{2}$ test for association. Genotypic $\chi^{2}$ tests for association were carried out as we had no a priori assumptions as to whether SNPs were acting under a specific mode of inheritance. Therefore, genotypic association tests were carried out to determine if any SNPs were acting dominantly or recessively to increase risk for alcohol dependence. We performed haplotype analysis to determine whether there are regions of KCNJ6 that harbor risk alleles that are inherited as haplotype blocks, which would not be detected by single-SNP association alone. To define these regions, we performed sliding window haplotype analysis.

Genotypes did not deviate significantly from HardyWeinberg equilibrium. Sliding window haplotype analysis was used to determine all haplotypes with a frequency greater than 0.05 . These were then tested for associations using the expectation maximization (EM) algorithm in the software packages PLINK v.1.04 (http://pngu.mgh.harvard. edu/ purcell/plink/) (Purcell et al, 2007b) and WHAP
(Purcell et al, 2007a). Permutation analysis of the haplotypic associations was conducted out to randomly re-assign the case and control labels in the data set and give a significance level corrected for all markers or haplotypes tested. A total of 10000 permutations were performed in each permutation analysis.

To examine gene-environment effects on adolescent hazardous drinking, linear regression analyses were performed with sex as a covariate. Models were fit for the main effects of the rs2836016 genotype and early and current psychosocial adversity with subsequent addition of the interaction term. Genotype groups did not differ with regard to sex, age, IQ, and psychosocial adversity (data not shown).

\section{In Silico Analysis}

Analysis of putative transcription factor binding sites was analyzed using MatInspector, Genomatix software, Munich, Germany (www.genomatix.de). Analysis of exon splicing efficiency was carried out using ACESCAN2 (http://genes. mit.edu/acescan2/index.html).

\section{RESULTS}

\section{SNP Selection and Gene Coverage}

KCNJ6 spans a large region of $300 \mathrm{~kb}$ on chromosome 21, and for this reason our genotyping efforts were concentrated on the $5^{\prime}$ region of the gene. We defined this region to include the KCNJ6 promoter as it extends from $10 \mathrm{~kb}$ upstream of the transcription start site and $80 \mathrm{~kb}$ into the gene to the genomic region of exon 2, where translation of the gene begins. SNPs were chosen firstly to maximize coverage of the promoter region and secondly to enrich for functional relevance, as the genomic regions surrounding the promoter and translation start sites may contain regulatory elements. Eleven SNPs were selected for genotyping (providing $82 \%$ coverage of the promoter region at $\left.r^{2}>0.8\right)$ and the relative positions of the SNPs chosen for genotyping and their linkage disequilibrium patterns are shown in Figure 1.

\section{Identification of Risk Genotypes for Alcoholism}

We analyzed 11 SNPs in KCNJ6 for their allelic association with alcoholism. An association was found with the SNP located at the intron/exon boundary of exon 2, rs 2836016 , and alcoholism in our sample of 1152 alcoholics vs 1203 controls $\left(\chi^{2}=9.73\right.$, FDR $p=0.01, \quad$ OR $\left.=1.2\right) \quad($ Table 1$)$, with $\mathrm{G}$ being the risk allele. Genotypic associations with alcoholism were also carried out to reveal SNP associations acting under a specific genetic mode of inheritance. These analyses revealed two promoter SNPs (rs2836048 and rs 2836049) to be nominally associated with alcoholism $\left(\chi^{2}=7.49, p=0.02\right.$ and $\chi^{2}=5.87, p=0.05$, respectively) (Supplementary Table S2). Genotype frequencies were in Hardy-Weinberg equilibrium (data not shown). Sliding window haplotype analyses found haplotype association with alcoholism in the genomic region of KCNJ6 extending from rs 283015 to rs2836049. A three SNP haplotype containing rs2836016, rs2226360, and rs 2836048 is associated with 


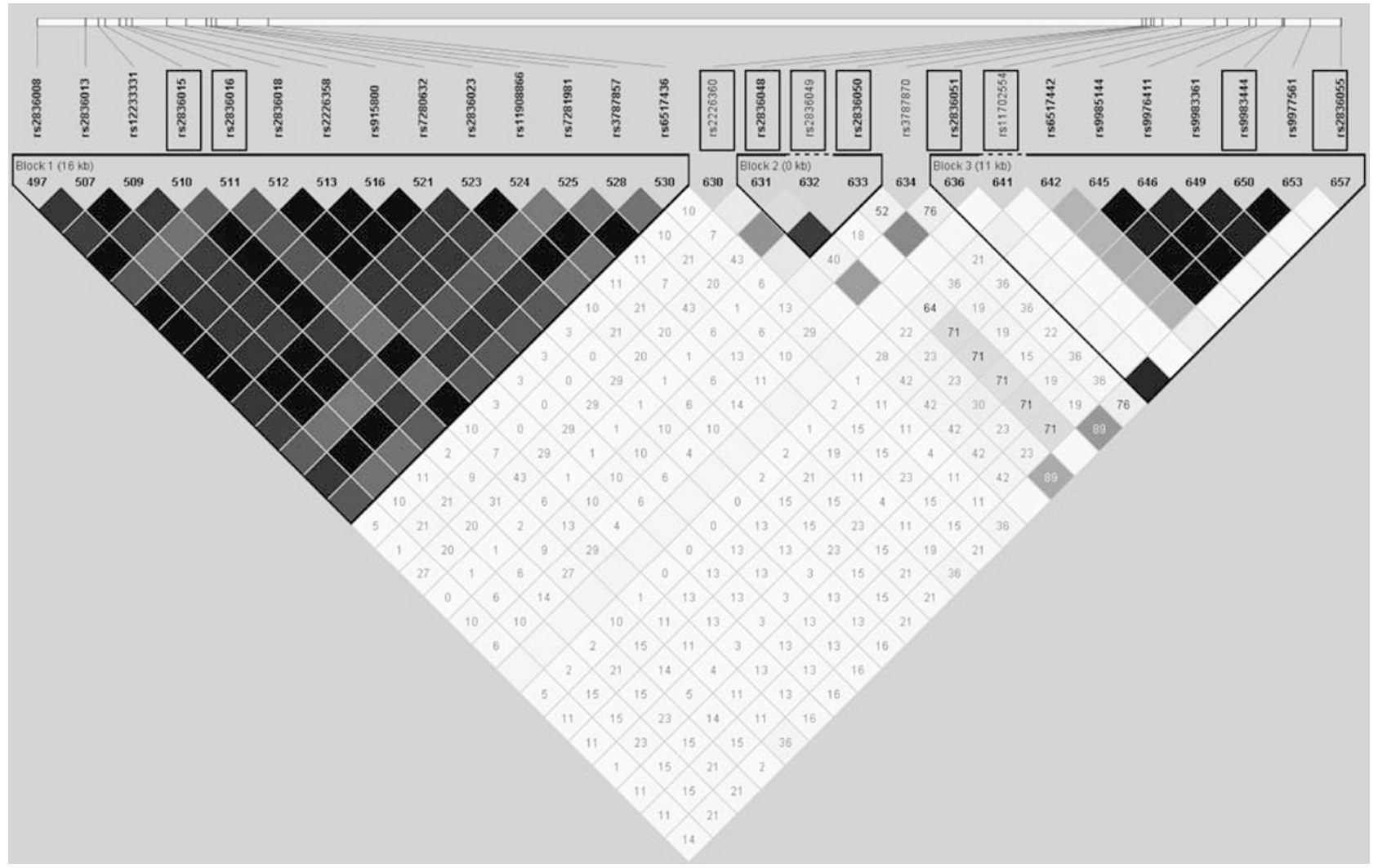

Figure I The relative positions of the SNPs chosen for genotyping, and their proxies. Genotyped SNPs are outlined in boxes. The numbers inside the linkage disequilibrium (LD) map represent $D^{\prime}$, and where $D^{\prime}$ is not shown, it can be taken to be I. Shading indicates a measure of $r^{2}$, where white represents $r^{2}=0$ and black represents $r^{2}=1$. LD blocks are defined according to the method identified by Gabriel et al (2002) in HAPLOVIEW vI.I Barrett et al (2005).

Table I Allelic Associations of I I SNPs Genotyped in KCNJ6, with the MAF of Cases and Controls Shown, Along with $\chi^{2}$ Values, Raw $p$-Values, FDR $p$-Values, and the OR

\begin{tabular}{|c|c|c|c|c|c|c|c|c|}
\hline SNP & Location & MAF cases & MAF controls & Minor allele & $\chi^{2}$ & $p$-Value & FDR & OR \\
\hline rs28360I5 & Intron & 0.3819 & 0.3991 & C & 1.34 & 0.247 & 0.5807 & 0.9303 \\
\hline rs 2836016 & Exon/intron boundary & 0.4861 & 0.4387 & G & 9.73 & 0.001813 & 0.01309 & 1.21 \\
\hline rs2226360 & Promoter & 0.02843 & 0.03103 & $\mathrm{~T}$ & 0.25 & 0.6198 & 0.6715 & 0.9137 \\
\hline rs2836048 & Promoter & 0.2396 & 0.2572 & A & 1.83 & 0.1758 & 0.5807 & 0.9097 \\
\hline rs2836049 & Promoter & 0.0879 & 0.07962 & $\mathrm{~T}$ & 0.98 & 0.3211 & 0.5807 & 1.114 \\
\hline rs|l 702554 & Promoter & 0.00726 & $0.007 \mid 75$ & G & 0.001 & 0.9736 & 0.9736 & 1.012 \\
\hline rs65।7442 & Promoter & 0.3173 & 0.3264 & C & 0.42 & 0.5189 & 0.6675 & 0.9593 \\
\hline rs9983444 & Promoter & 0.224 & 0.211 & C & 1.1 & 0.2944 & 0.5807 & 1.079 \\
\hline rs2836055 & Promoter & 0.106 & 0.1131 & C & 0.58 & 0.4488 & 0.6482 & 0.9291 \\
\hline
\end{tabular}

Abbreviations: FDR, false discovery rate; MAP, minor allele frequencies; OR, odds ratios.

FDR significant results are highlighted in bold.

alcoholism with the AGA haplotype being significantly over-represented in controls $(p=0.001)$ (Table 2). When this haplotype was analyzed with rs2836016 dropped, the association no longer remains significant (omnibus test $p$-value $=0.21)$. This suggests that the haplotypic association is driven by the SNP rs2836016. Therefore, we selected rs 2836016 for subsequent analyses.

\section{Gene-Environment Interaction}

The frequency of the rs 2836016 minor $(\mathrm{G})$ in the adolescent sample is comparable to the frequency in the adult control sample (shown in Supplementary Table S3). The distribution of genotypes was in accordance with Hardy-Weinberg equilibrium $(p>0.50)$. Regression results 
Table 2 Significant Haplotype Associations Identified Using a Sliding Window Analysis, Between KCNJ6 and Alcohol Drinking in the Adult Sample of I 152 Cases vs 1203 Controls

\begin{tabular}{|c|c|c|c|c|c|c|c|c|}
\hline rs28360I5 & rs2836016 & rs2226360 & rs2836048 & rs2836049 & Global P & \% Cases & $\%$ Controls & p-Value \\
\hline $\mathrm{T}$ & G & & & & 0.009 & 0.4863 & 0.4442 & 0.007974 \\
\hline \multirow[t]{3}{*}{$\mathrm{T}$} & A & & & & & 0.1342 & 0.1614 & 0.0163 \\
\hline & A & G & & & & 0.5129 & 0.5521 & 0.01445 \\
\hline & & & A & C & 0.03373 & 0.1498 & 0.1791 & 0.01192 \\
\hline & A & G & A & & 0.004 & 0.09577 & 0.1268 & 0.001109 \\
\hline & G & G & G & & & 0.3464 & 0.3138 & 0.02145 \\
\hline C & A & G & A & & 0.008 & 0.07575 & 0.09818 & 0.008786 \\
\hline $\mathrm{T}$ & G & G & G & & & 0.3453 & 0.3129 & 0.02225 \\
\hline
\end{tabular}

Global p-values for haplotype tests and individual p-values for each haplotype are presented. Bold highlighted individual $p$-values remain significant after 10000 permutations

Table 3 Multiple Linear Regression Models Testing the Effects of rs2836016 Genotype, Early and Current Psychosocial Adversity, and Their Interaction on Hazardous Drinking in Adolescents ( $\beta$ Co-efficients and Raw p-Values are Shown)

\begin{tabular}{|c|c|c|c|c|c|c|}
\hline & \multicolumn{2}{|c|}{$\begin{array}{l}\text { Rs2836016 } \\
\text { genotype }\end{array}$} & \multicolumn{2}{|c|}{$\begin{array}{l}\text { Psychosocial } \\
\text { adversity }\end{array}$} & \multicolumn{2}{|c|}{$\begin{array}{c}\text { Rs } 2836016 \times \\
\text { psychosocial } \\
\text { adversity }\end{array}$} \\
\hline & $\beta$ & p-Value & $\beta$ & $p$-Value & $\beta$ & p-Value \\
\hline \multicolumn{7}{|c|}{ Early family adversity } \\
\hline AUDIT score & 0.029 & 0.505 & 0.15 & 0.01 & 0.427 & 0.014 \\
\hline \multicolumn{7}{|c|}{ Current stressful life events } \\
\hline AUDIT score & -0.005 & 0.933 & 0.356 & $<0.001$ & 0.353 & 0.066 \\
\hline
\end{tabular}

revealed a significant interaction between rs2836016 and early psychosocial adversity on hazardous drinking in 19-year olds (see Table 3). Subsequent analysis showed that the number of early adversity factors present was associated with increasing AUDIT scores among G homozygotes $(\beta=0.352, p=0.004)$, but not among AG carriers $(\beta=-0.109, \quad p=0.205)$ and $\mathrm{A}$ homozygotes $(\beta=0.029$, $p=0.780)$. Figure 2 illustrates the interaction, indicating higher AUDIT scores in GG carriers exposed to high adversity compared with all other groups. For this analysis, individuals were grouped according to a median split on the early adversity score. Furthermore, significant main effects of psychosocial adversity on hazardous drinking in 19-year olds emerged, but no significant main effect of rs2836016 (Table 3).

The adolescent sample had also been characterized for current stressful life events, in the 4 years before the 19-year assessment. Regression results of the analysis of current stress and rs2836016 genotype on AUDIT scores revealed

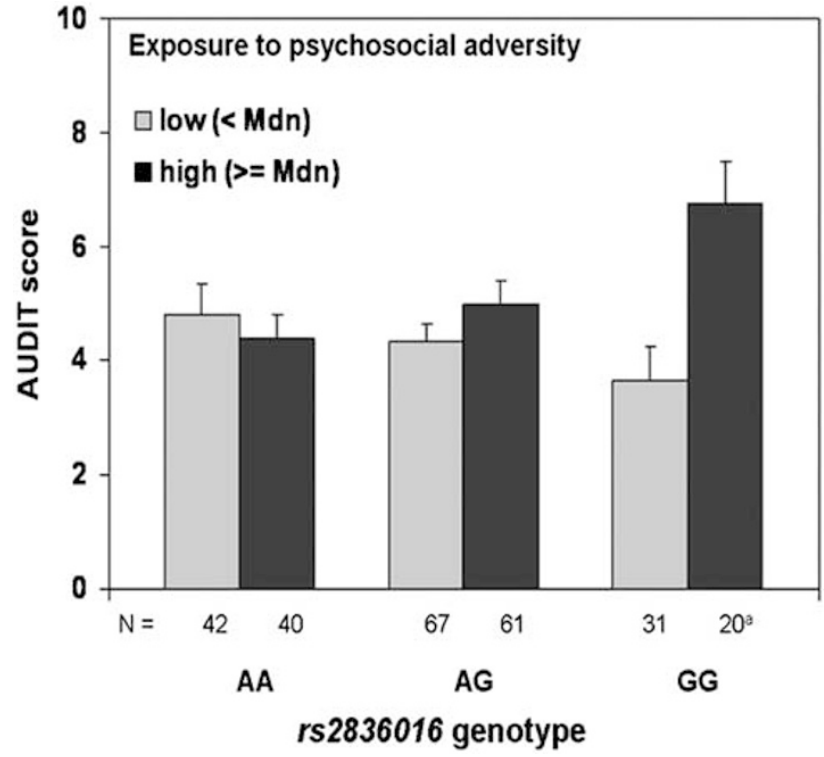

Figure 2 Mean scores (and SE) on the AUDIT in adolescents grouped by rs2836016 genotype and exposure to early psychosocial adversity $N=$ number of individuals. ${ }^{a}$ Significantly different from all other groups according to Fisher's LSD.

similar results; however, the gene-environment interaction was not statistically significant (Table 3). (Means and standard deviations for AUDIT scores, early psychosocial adversity, and current stressful life events are shown in Supplementary Table S4.)

\section{Analysis of Nicotine Dependence}

Data on nicotine dependence was available for 970 individuals in the alcohol-dependent cohort. Sixty-six percent of the adult alcohol dependents also met the diagnostic criteria of nicotine dependence and $18 \%$ were classed as 
non-smokers. The three SNPs that had previously been shown to be associated with alcoholism were analyzed by performing association analysis in the alcohol-dependent cohort comparing nicotine dependents and non-smokers. None of the three SNPs associated with alcohol dependence were associated with nicotine dependence in the alcoholic cohort $(p \geqslant 0.102)$ (Supplementary Table S5).

\section{DISCUSSION}

We report an association of the KCNJ6 polymorphism, rs2836016, with alcoholism in an adult alcohol-dependent sample. Genotypic associations revealed nominal associations with two promoter SNPs: rs2836048 and rs2836049. Haplotype analysis revealed a three SNP haplotype consisting of rs2836016, rs2236360, and rs2836048 (AGA), which is protective against alcohol dependence as it was found to be at a significantly higher frequency in the adult non-alcoholdependent controls. As rs2836016 was found to have the strongest association with adult alcohol dependence and formed part of a significant haplotype that increased risk for alcohol dependence, this SNP was carried forward to be examined in an adolescent sample characterized for early life stress and drinking behavior. KCNJ6 may also be associated with the initiation of alcohol abuse as we find a gene $\times$ environment interaction with rs2836016 and psychosocial adversity in adolescents whereby adolescent carriers of the $G$ allele who experience high psychosocial adversity show higher AUDIT scores.

Early life stress has been shown to increase the propensity for alcohol consumption in later life, as neonatal separation paradigms in Rhesus macaques have shown (Fahlke et al, 2000). Although there is currently no evidence to suggest a mechanism by which early life stress alters alcohol consumption in later life, the data presented herein suggest that polymorphisms in KCNJ6 may mediate such effects. GIRK2 channels have been shown to interact with neuronal stress systems including the CRH system (Beckstead et al, 2009), and our results show that polymorphisms in KCNJ6 associate with increased risky drinking in adolescents when early psychosocial stress is present. Furthermore, these polymorphisms are also relevant for adult alcoholism, suggesting that the interaction between early psychosocial stress and KCNJ6 risk genotypes may confer risk to alcoholism that persists until adulthood.

Using an animal model, Song et al (2007) found that it was not possible to establish a place preference for ethanol in adolescent rats unless they were exposed to chronic stress, suggesting that stress enhances the rewarding effects of ethanol in adolescence. Increased reinforcement for alcohol drinking during adolescence may enhance the potential for alcohol abuse in later life. The interaction between GIRK2 and neurobiological stress systems is suggestive of a mechanism by which the reinforcing effects of alcohol develop during periods of environmental stress. Indeed, Hill et al (2003) found that they were unable to establish a place preference for ethanol in GIRK2-null mice, which confirms the notion that GIRK2 is important for alcohol reward. In support of this, we find that genetic variation in GIRK2 is associated with the initiation of alcohol abuse in adolescents when psychosocial stress is present, and in addition, an association is also found with the maintenance of alcohol dependence in adults.

Given the importance of GIRK2 in dopaminergic neurotransmission and the interaction of GIRK2 with neuronal stress systems, we propose that GIRK2 mediates the effects of psychosocial stress on dopaminergic signalling. This is supported by the evidence that GIRK2 levels are affected by cortisol in the rat brain (Muma and Beck, 1999) and that CRF enhances the efficacy of the GIRK channels in dopaminergic neurons (Beckstead et al, 2009). The effects of psychosocial stress may disrupt dopaminergic reward circuitry in the brain and in turn affect alcohol drinking behavior. This hypothesis is supported by our data as we find that genetic variation in GIRK2 is associated with alcohol abuse in adolescents, but only when psychosocial stress is present. This finding provides further support that GIRK2 mediates the effects of stress on dopaminergic signaling, and that this alters alcohol drinking owing to the disruption of neuronal reward systems.

Previous studies of KCNJ6 in human beings have shown that it is not only relevant for alcohol dependence, but also addiction to nicotine. Saccone et al (2007) found that SNP rs651772 in the promoter of KCNJ6 was among the top hits for nicotine dependence when analyzing over 3000 SNPs across nicotine-addiction candidate genes. It should be noted that $62 \%$ of our adult alcohol-dependent sample are also addicted to nicotine. The nicotine-addicted population of the alcohol-dependent sample was analyzed separately and compared with the non-nicotine-addicted alcoholics to determine if these individuals were driving our association with KCNJ6. For all three SNPs initially associated with alcoholism, none remained significant when this analysis was carried out, which suggests that the association of these SNPs reflects that of alcohol dependence.

In silico analysis was carried out to elucidate the potential biological role of rs2836016 in influencing alcoholism. The analysis revealed that the A allele of rs2836016 completely abolishes the binding site of SRp40, a known splicing factor, thus potentially disrupting the splicing efficiency of KCNJ6. Inanobe et al (1999) report that dopaminergic neurons were found to be regulated by channels formed of different GIRK2 splice variant subunits in the substantia nigra (SN). They found that GIRK2 channels composed of different combinations of GIRK2 splice variants displayed different levels of G-protein stimulation. If rs2836016 does indeed affect the splicing efficiency of KCNJ6, it may contribute to the relative levels of GIRK2 isoforms in the brain, and thus have an effect on the rate of dopaminergic signalling in the $\mathrm{SN}$, which in turn would affect alcohol drinking behavior. Considering the role of GIRK2 in dopaminergic signalling, this association may reflect individuals consuming more alcohol to experience its rewarding effects.

In conclusion, our data provide the first evidence of an association of KCNJ6 with alcoholism in human beings. We propose that KCNJ6 moderates the effects of psychosocial stress in adolescents by disrupting dopaminergic signalling and in turn this affects the rewarding effects of alcohol, predisposing individuals to initiate alcohol use later in life. The effects of cortisol on GIRK2 levels in the brain suggest that stress may serve to reinforce the rewarding properties of alcohol. Further work would include an analysis of GIRK2 in other alcoholic populations to replicate this 
finding and studies of KCNJ6 alcohol and nicotine comorbidity cohorts would also be of interest. In addition, in vitro analysis would help gauge the functional role of the associated SNPs (or their tag SNPs) in GIRK2 expression and splicing efficiency, to elucidate the functional mechanism by which these SNPs confer vulnerability to alcohol abuse.

\section{ACKNOWLEDGEMENTS}

This work was supported by the European Commission FP-6 integrated project IMAGEN (PLO37286) and coordinated project ADAMS (242257), as well as the UK-NIHRBiomedical Research Centre Mental Health, the MRCAddiction Research Cluster 'Genomic Biomarkers', and the MRC program grant 'Developmental pathways into adolescent substance abuse' (93558). This work was also supported by the BMBF Grant FKZ EB 01011300, NGFN2 Grant FKZ01GS0117, and DFG Grant LA 733/1-2 et al, and additional BMBF Grant support in the framework of NGFN2.

\section{DISCLOSURE}

The authors TKC, ML, NW, MR, WM, ML, AL, UZ, SD, and GS report no biomedical financial interests or potential conflicts of interest.

\section{REFERENCES}

Barrett JC, Fry B, Maller J, Daly MJ (2005). Haploview: analysis and visualization of LD and haplotype maps. Bioinformatics (Oxford, England) 21: 263-265.

Beckstead MJ, Gantz SC, Ford CP, Stenzel-Poore MP, Phillips PE, Mark GP et al (2009). CRF enhancement of GIRK channelmediated transmission in dopamine neurons. Neuropsychopharmacology 34: 1926-1935.

Benjamini Y, Drai D, Elmer G, Kafkafi N, Golani I (2001). Controlling the false discovery rate in behavior genetics research. Behav Brain Res 125: 279-284.

Bohn MJ, Babor TF, Kranzler HR (1995). The Alcohol Use Disorders Identification Test (AUDIT): validation of a screening instrument for use in medical settings. J Stud Alcohol 56: 423-432.

Dick DM, Bierut LJ (2006). The genetics of alcohol dependence. Curr Psychiatry Rep 8: 151-157.

Dube SR, Miller JW, Brown DW, Giles WH, Felitti VJ, Dong M et al (2006). Adverse childhood experiences and the association with ever using alcohol and initiating alcohol use during adolescence. J Adolesc Health 38: 444.e441-410.

Dybek I, Bischof G, Grothues J, Reinhardt S, Meyer C, Hapke U et al (2006). The reliability and validity of the Alcohol Use Disorders Identification Test (AUDIT) in a German general practice population sample. J Stud Alcohol 67: 473-481.

Fagerstrom KO (1978). Measuring degree of physical dependence to tobacco smoking with reference to individualization of treatment. Addict Behav 3: 235-241.

Fahlke C, Lorenz JG, Long J, Champoux M, Suomi SJ, Higley JD (2000). Rearing experiences and stress-induced plasma cortisol as early risk factors for excessive alcohol consumption in nonhuman primates. Alcohol Clin Exp Res 24: 644-650.
Gabriel SB, Schaffner SF, Nguyen H, Moore JM, Roy J, Biumenstiel $\mathrm{B}$ et al (2002). The structure of haplotype bioces in the human genome. Science 296: 2225-2229.

Grimm D (2008). Public health. Staggering toward a global strategy on alcohol abuse. Science (New York, NY) 16: 862-863.

Hill KG, Alva H, Blednov YA, Cunningham CL (2003). Reduced ethanol-induced conditioned taste aversion and conditioned place preference in GIRK2 null mutant mice. Psychopharmacology 169: 108-114.

Inanobe A, Yoshimoto Y, Horio Y, Morishige KI, Hibino $\mathrm{H}$, Matsumoto S et al (1999). Characterization of G-protein-gated $\mathrm{K}^{+}$channels composed of Kir3.2 subunits in dopaminergic neurons of the substantia nigra. J Neurosci 19: 1006-1017.

Kuhner C (1997). Fragebogen zur Depressionsdiagnostik nach DSM-IV (FDD-DSM-IV). Hogrefe.

Kuzhikandathil EV, Yu W, Oxford GS (1998). Human dopamine D3 and D2L receptors couple to inward rectifier potassium channels in mammalian cell lines. Mol Cell Neurosci 12: 390-402.

Laucht M, Esser G, Baving L, Gerhold M, Hoesch I, Ihle W et al (2000). Behavioral sequelae of perinatal insults and early family adversity at 8 years of age. J Am Acad Child Adolesc Psychiatry 39: 1229-1237.

Laucht M, Esser G, Schmidt MH (1997). Developmental outcome of infants born with biological and psychosocial risks. J Child Psychol Psychiatry Allied Discipl 38: 843-853.

Muma NA, Beck SG (1999). Corticosteroids alter G protein inwardly rectifying potassium channels protein levels in hippocampal subfields. Brain Res 839: 331-335.

Nicoll RA (1988). The coupling of neurotransmitter receptors to ion channels in the brain. Science (New York, NY) 241: 545-551.

Purcell S, Daly MJ, Sham PC (2007a). WHAP: haplotype-based association analysis. Bioinformatics (Oxford, England) 23: 255-256.

Purcell S, Neale B, Todd-Brown K, Thomas L, Ferreira MA, Bender D et al (2007b). PLINK: a tool set for whole-genome association and population-based linkage analyses. Am J Hum Genet 81: 559-575.

Robbins TW, Everitt BJ (1999). Drug addiction: bad habits add up. Nature 398: 567-570.

Rutter MQ, Quinton D (1977). Psychiatric disorder-ecological factors and concepts of causation. In: M McGurk (ed) Ecological Factors in Human Development. Elsevier: NorthHolland, Amsterdam, pp 173-187.

Saccone SF, Hinrichs AL, Saccone NL, Chase GA, Konvicka K, Madden PA et al (2007). Cholinergic nicotinic receptor genes implicated in a nicotine dependence association study targeting 348 candidate genes with 3713 SNPs. Hum Mol Genet 16: 36-49.

Song M, Wang XY, Zhao M, Wang XY, Zhai HF, Lu L (2007). Role of stress in acquisition of alcohol-conditioned place preference in adolescent and adult mice. Alcohol Clin Exp Res 31: 2001-2005.

Tupala E, Tiihonen J (2004). Dopamine and alcoholism: neurobiological basis of ethanol abuse. Prog Neuro-Psychopharmacol Biol Psychiatry 28: 1221-1247.

Werner P, Hussy N, Buell G, Jones KA, North RA (1996). D2, D3, and D4 dopamine receptors couple to $G$ protein-regulated potassium channels in Xenopus oocytes. Mol Pharmacol 49: 656-661.

Wittchen HU, Essau CA, Hecht H, Teder W, Pfister H (1989). Reliability of life event assessments: test-retest reliability and fall-off effects of the Munich Interview for the Assessment of Life Events and Conditions. J Affect Disord 16: 77-91.

Wittchen HU, Zhao S, Abelson JM, Abelson JL, Kessler RC (1996). Reliability and procedural validity of UM-CIDI DSM-III-R phobic disorders. Psychol Med 26: 1169-1177.

Supplementary Information accompanies the paper on the Neuropsychopharmacology website (http://www.nature.com/npp) 